\title{
Osteopenia-intellectual disability-sparse hair syndrome
}

INSERM

\section{Source}

INSERM. (1999). Orphanet: an online rare disease and orphan drug data base.

Osteopenia-intellectual disability-sparse hair syndrome. ORPHA:2324

Kaler-Garrity-Stern syndrome is a rare syndrome, described in two sisters of Mennonite descent, characterized by sparse hair, osteopenia, intellectual disability, minor facial abnormalities, joint laxity and hypotonia. There have been no further descriptions in the literature since 1992. 